\title{
Supporting Information: Simpler is Better: How Linear Prediction Tasks Improve Transfer Learning in Chemical Autoencoders
}

\author{
Nicolae C. Iovanac and Brett M. Savoie*
}

Charles D. Davidson School of Chemical Engineering, 480 Stadium Mall Drive, Purdue

University, West Lafayette, IN 47906, USA.

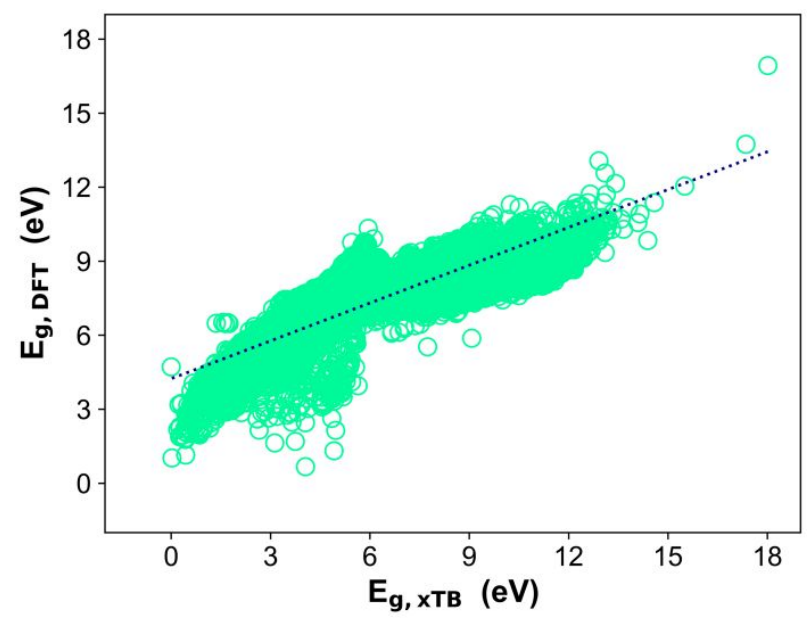

Figure S1: Correlation plot between bandgap calculated at the DFT and xTB levels of theory $\left(\mathrm{R}^{2}=0.78\right) . E_{g, x T B}$ systematically overestimates the bandgap compared with $E_{g, D F T .}$. All values are positive. Utilizing a linear regression on all available DFT and XTB training data results in a MAE in predicting the DFT bandgap of the test set of $0.47 \mathrm{eV}$. 


\section{Model Architecture}

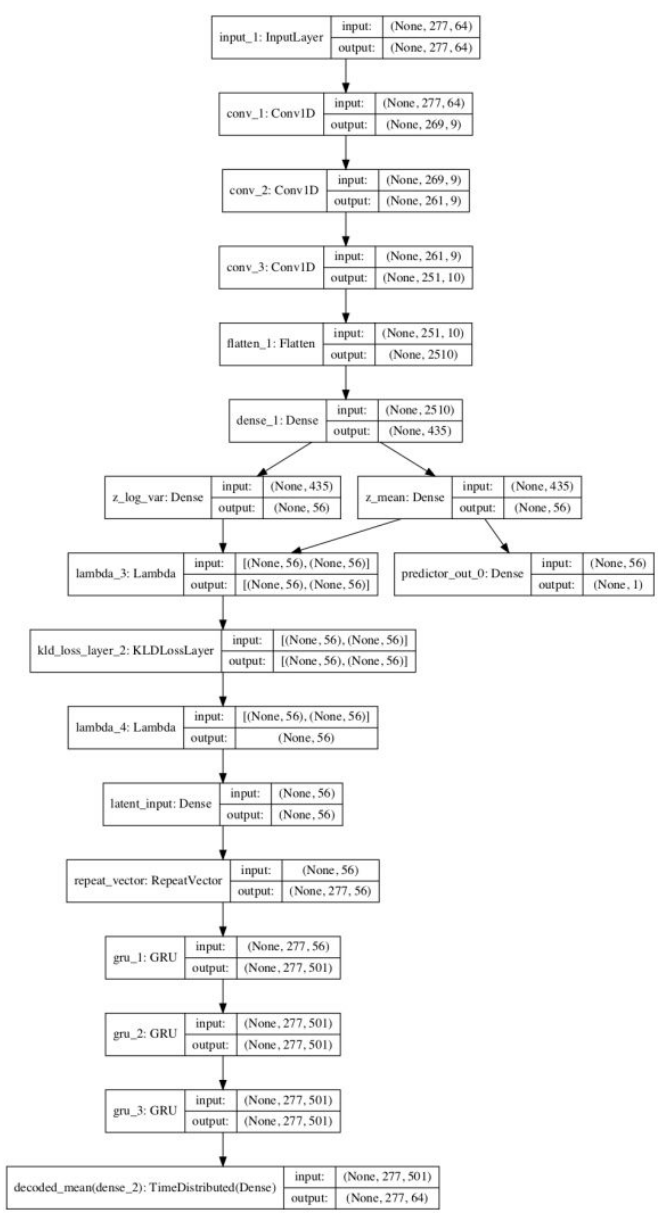

Figure S2. Variational autoencoder architecture utilized for property prediction and latent space analysis as implemented in Keras 2.2.4 with Tensorflow 1.14.0 backend. 


\section{Multi-property Prediction PCA Plots}

A

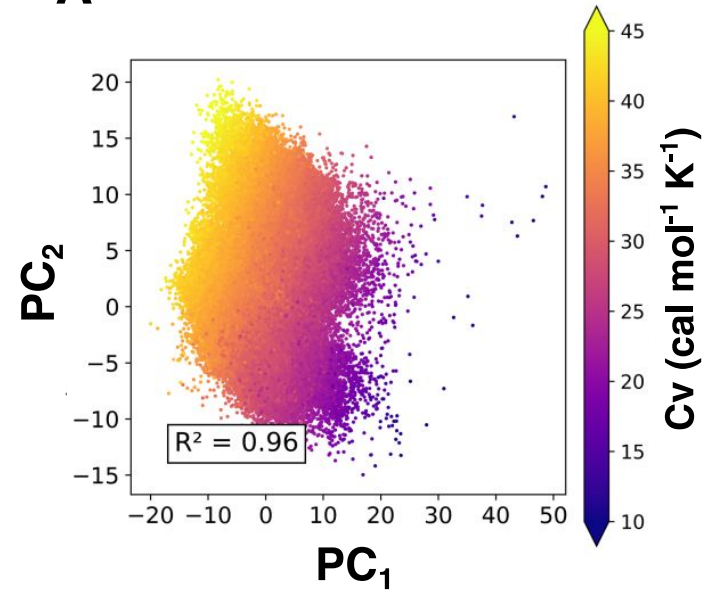

B

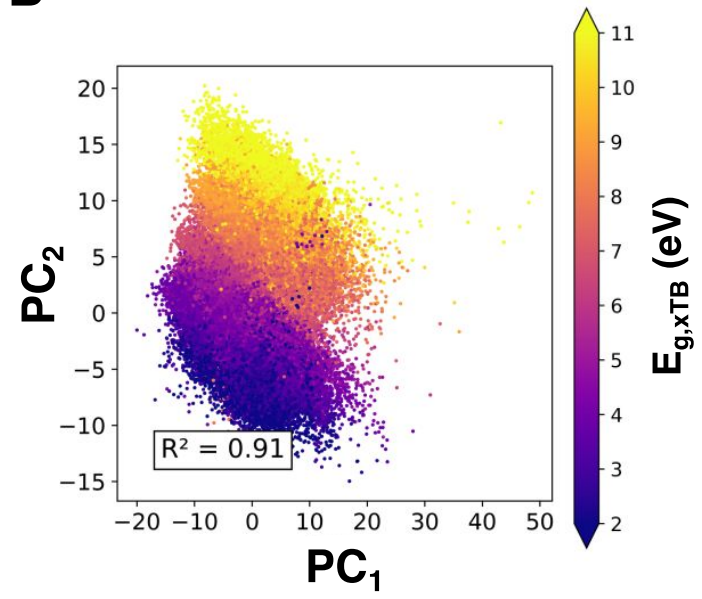

Figure S3: Principal component analysis performed on latent encodings for entire QM9 dataset for a model trained on both XTB data and heat capacity with a predictor network consisting of a single linear node. Projections are colored according to (A) $C v$ and (B) $E_{g, x T B}$. In contrast to the more complex predictor network utilized in Figure S4, the linear network is capable of organizing multiple properties linearly and along orthogonal directions within the latent space
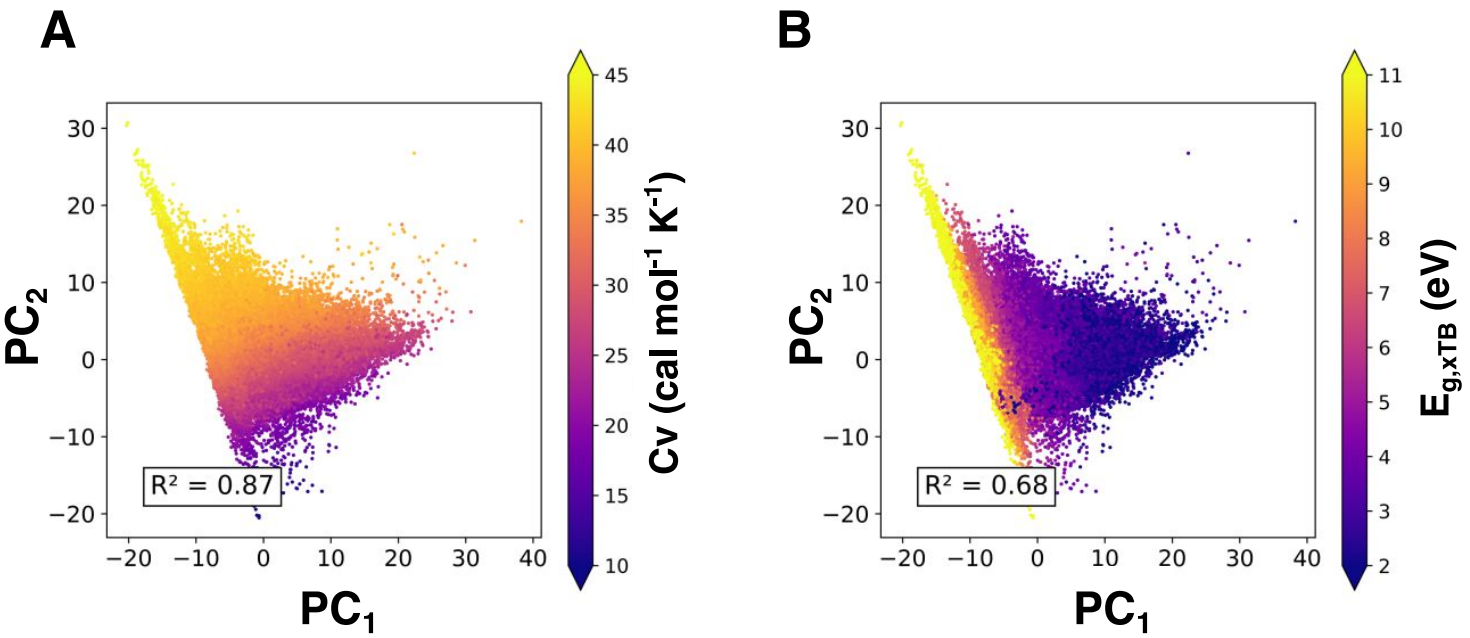

Figure S4: Principal component analysis performed on latent encodings for entire QM9 dataset for a model trained on both xTB data and heat capacity with a predictor network consisting of $3 \times 64$ nodes. Projections are colored according to (A) $C_{v}$ and (B) $E_{g, x T B}$. While the latent space is well organized in a linear fashion according to $C_{v}$, the organization with respect to $E_{g, x T B}$ is poorly organized. Rather than varying linearly, the projections suddenly ramp up to very high $E_{g, x T B}$ structures. 